\section{Cardiopulmonary resuscitation in the elderly: analysis of the events in the emergency department}

\author{
Augusto Tricerri,, Lorenzo Palleschi, \\ Luigi Zulli' \\ 'Emergency Department, S. Filippo Neri \\ Hospital, Roma; ${ }^{2}$ Emergency and Internal \\ Medicine Department, S. Giovanni \\ Addolorata Hospital, Rome, Italy
}

\section{Abstract}

With the increasing number of old people in all western countries and increasing life expectancy at birth, many seniors spend the last period of their life with various afflictions that may lead to cardiac arrest. Bystander cardiopulmonary resuscitation (CPR) increases survival rates. Octogenarians are the fastest growing segment of the population and despite empirical evidence that CPR is of questionable effectiveness in seniors with comorbidities, it is still the only treatment among lifesustaining ones. Cardiopulmonary resuscitation is frequently unsuccessful, but if survival is achieved, a fairly good quality of life can be expected. Various papers analyzed the effect of CPR in hospitalized patients or in cardiac arrest occurring at home or in public places, while less is known about events occurring in the emergency room (ER). We performed a retrospective analysis of cardiac arrest events occurred in ER within 54 months: we analyzed 415,001 records of ER visits (from 01/01/1999 to $30 / 06 / 2003$ ) in San Giovanni Addolorata Hospital. Data were analyzed in terms of age and outcome. We identified 475 records with the outcome of death in ER or death on arrival. Out of them, we selected 290 medical records which had sufficient data to be analyzed. Of the 290 patients evaluated, 225 died in ER, 18 were deemed to die on arrival, and 47 survived the cardiac arrest and were admitted to intensive care unit (ICU). The overall mortality was $0.11 \%$, while the incidence of the selected events was $0.072 \%$. The mean age of the analyzed population was 71.3 years. The only possible diagnosis was often cardiac arrest, though most of the times we could specify and group the diagnosis even better. The analysis of the procedures showed that cardiac arrest treated by direct current (DC) shock was similarly distributed in different age groups, and no difference was detectable between the two groups. The mean age of the patients who underwent tracheal intubation (TI) was slightly but significantly lower in comparison with the group of non-intubated patients, thus showing a less aggressive resuscitator behavior in old people. Old people seem to have the same chance for surviving cardiac arrest as young people. Other factors like comorbidity can influence the outcome of a cardiac arrest in the elderly. The results of the present study demonstrate that age itself does not seem to be an independent, unfavorable prognostic factor for the outcome after CPR and that older people must be offered the same chance for surviving cardiac arrest as youngsters.

\section{Introduction}

With the increasing number of old people in all western countries and the increasing life expectancy at birth, many seniors spend the last period of their life with various afflictions that may lead to cardiac arrest. Bystander cardiopulmonary resuscitation (CPR) increases survival rates; however, the largest group of cardiac arrest patients are over 60 men being at home, whose most probable potential CPR provider is an old woman not likely to have received CPR training. Despite empirical evidence that cardiopulmonary resuscitation (CPR) is of questionable effectiveness in seniors with comorbidities, it continues to hold a unique place in the armamentarium of life-sustaining treatments, and the consent for CPR implies that, when needed, it is administered emergently.

The use of life-sustaining treatments is prevalent in very old patients who die in the course of hospitalization or during institutionalization, despite the fact that the majority prefer comfort care. Indeed, during the last period of life, many seniors and their families face decisions that challenge ethical principles and may cause conflict among family members as well as healthcare professionals.

Evaluation of outcome after cardiac arrest focuses mainly on survival. Survivors of cardiac arrest end up in different states of health, and survival alone may not be a sensitive measure for successful cardiopulmonary resuscitation. Many reports have shown that CPR is frequently unsuccessful, but if survival is achieved a fairly good quality of life can be expected. $^{1}$

Age is thought not to be an adequate criterion to predict survival ${ }^{2}$ and there is limited evidence that functional status may be a predictor of poor CPR, ${ }^{3}$ as it was reported that individuals who had a stroke are less likely to survive CPR than others. ${ }^{4}$

Different papers analyzed the effect of cardiopulmonary resuscitation either in hospitalized patients, considering also the ethical issues of do-not-resuscitate (DNR) orders, or in people staying at home or being in public places. Less is known about the events occur-
Correspondence: Augusto Tricerri, Emergency Department, S. Filippo Neri Hospital, via Martinotti 20, 00135 Rome, Italy.

Tel. +39.06 .3306 .2387 - Fax: +39.06 .3306 .2584 .

E-mail: a.tricerri@sanfilipponeri.roma.it

Key words: emergency department, cardiopulmonary resuscitation, aging, advanced life support.

Contributions: the authors contributed equally.

Received for publication: 15 February 2013. Accepted for publication: 15 April 2013.

This work is licensed under a Creative Commons Attribution 3.0 License (by-nc 3.0).

(C) Copyright A. Tricerri et al., 2013

Licensee PAGEPress, Italy

Emergency Care Journal 2013; 9:e10

doi:10.4081/ecj.2013.e10

ring in the emergency room (ER).

Therefore, we performed a retrospective analysis of the cardiac arrest events occurred in ER during 54 months and analyzed the data according to patients' age and outcome.

\section{Materials and Methods}

In Lazio region (Italy) emergency departments and first aids in small hospitals all use the same software which generates a comprehensive medical record including clinical data reported by the physician and laboratory, and $\mathrm{X}$-ray reports. Using the database of this program (GIPSE; Sago Medica S.r.l., Pieve di Cento, Italy) we analyzed 415,001 records of ER visits occurring from 01/01/1999 to 30/06/2003 in one of the main hospitals of Rome - San Giovanni Addolorata Hospital. We identified 475 records with the outcome of death in the ER or death on arrival. After a check for the congruence between outcome and main diagnosis, 466 records were evaluated. We then ran a query in the record of the diagnosis searching for cardiac arrest or cardiopulmonary arrest or ventricular fibrillation and we were able to identify a set of 298 medical records, and of these 290 included sufficient data to be analyzed. For every patient we reported age, sex, undertaken procedures, drugs used, final diagnosis and outcome in ER. Then, we collected the data of the final outcome and the length of stay for the patients who survived and were admitted to the intensive care unit (ICU). For grouping purposes, we categorized the patients as young (i.e. age $0-65$ ) or aged (i.e. age $>65$ ).

The collected data were then evaluated by 
means of a statistic software (Statistica; StatSoft inc., Tulsa, OK, USA) using a descriptive statistic tool and the Student's t-test or Fisher's exact test when convenient.

\section{Results}

Of the 290 patients evaluated, 225 died in ER, 18 were deemed to die on arrival, and 47 (16.2\%) survived the cardiac arrest and were admitted to an ICU. The overall mortality resulted $0.11 \%$, while the selected events' incidence was $0.072 \%$. The mean age of the analyzed population was 71.3 years (range $0-96$ ) and the distribution is shown in Figure 1. The age ranges stratified for the outcome is shown in Table 1 and Figure 2. The rate of return of spontaneous circulation (ROSC) and survival did not differ significantly among the young and elderly patients (15 vs $16.7 \%$, and $6.25 \mathrm{vs}$ $6.19 \%)$. We made an effort to analyze the final diagnosis, as it was stated in the ER record, to assess the occurrence: as it could be expected, in a relevant number of cases the only possible diagnosis was cardiac arrest, but in many cases we were able to better specify and group the diagnosis (Figure 3). Among the remaining diagnoses, myocardial infarction, neurological diseases or coma, and pre-existing cardiomyopathy are the most frequent.

The mean age of the patients who underwent the procedure of tracheal intubation (TI) was slightly but significantly lower in comparison with the group of non-intubated ones showing a less aggressive resuscitator behavior in aged patients (Table 2, Figure 4).

The analysis of the procedures showed that the cardiac arrest treated by direct current (DC) shock was similarly distributed in different age groups and that no difference was detectable between the two groups (Figure 5). Furthermore, we were able to collect some data about the outcome and the hospital stay of the admitted patients from the administrative hospital discharge records. Six out of the 47 patients were transferred to another hospital (13\%), whereas 23 died (49\%) and 18 were discharged from the hospital (38\%) (Figure 6).

The mean hospital stay for the deceased patients was 8.4 days and for the discharged ones 15.44 (range 1-80 and 2-68, respectively; $\mathrm{P}=0.16306$, two tailed unpaired Student's $\mathrm{t}$ test). After grouping the admitted patients according to age, we analyzed the survival rate among young and aged patients that resulted 50 and $41.9 \%$, respectively ( $\mathrm{P}=$ not significant; Fisher's exact test). The mean survival time among young patients resulted longer than in aged ones, although it did not reach the statistical significance (deceased $17.4 \pm 35$ vs $7.4 \pm 6.9 ; \mathrm{P}=$ not significant, two tailed unpaired Student's t test) and also hospital staying of discharged patients resulted longer in young than in aged subjects, without reaching statistical significance $(26.6 \pm 24.8$ vs $11.15 \pm 5.969$; $\mathrm{P}=$ not significant, two tailed unpaired Student's t-test) (Figure 7).

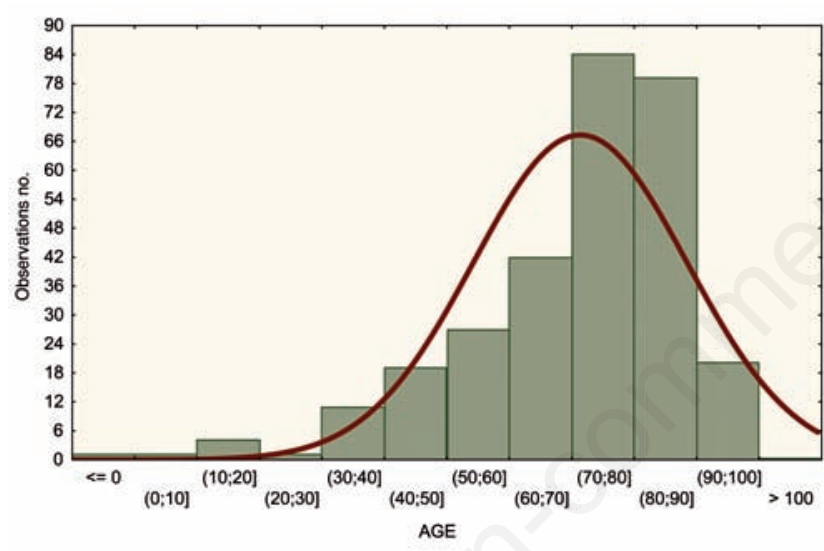

Figure 1. Age distribution of the selected population.

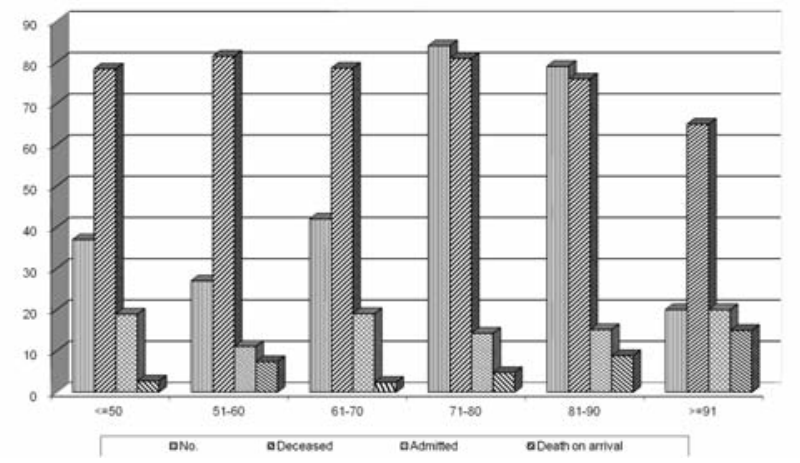

Figure 2. Age distribution according to the outcome.
Table 1. Age analysis according to the outcome.

\begin{tabular}{lccc} 
& Minimum & Maximum & Mean \\
Admitted to hospital & 3 & 96 & 71.73 \\
Deceased & 0 & 95 & 71.04 \\
\hline Death on arrival & 19 & 95 & 76.06 \\
Total & 0 & 96 & 71.46 \\
\hline
\end{tabular}

Table 2. Age of patients who underwent tracheal intubation or not compared by means of the Student's t test.

\begin{tabular}{lccccc} 
& $\begin{array}{c}\text { Groulp 1 - NII } \\
\text { (mean) }\end{array}$ & $\begin{array}{c}\text { Group 2 - TI } \\
\text { (mean) }\end{array}$ & t & df & P \\
Age & 73.7755 & 69.1343 & 2.302503 & 279 & 0.022043 \\
\hline NTI, not tracheally intubated; TI, tracheally intubated; df, degree of freedom. & &
\end{tabular}

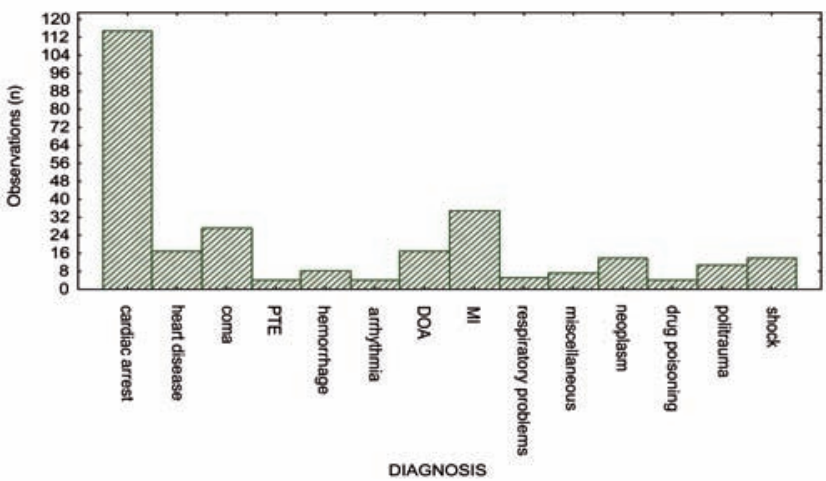

Figure 3. Main diagnosis in cardiac arrest patients. PTE, pulmonary thromboembolism; DOA, death on arrival; MI, myocardial infarction. 


\section{Discussion}

In the last decades, old people have increased in western countries, and although comorbidity and disability are still important factors limiting quality of life, the successful ageing of most of these subjects opens new perspectives in the care of sudden cardiac arrest too. Moreover, it was reported that the proportion of out-of-hospital cardiac arrest (OHCA) events occurring at nursing homes increased with advancing age. ${ }^{5}$

Death for sudden cardiac arrest occurs in 350,000 to 450,000 patients per year in the USA alone and $90 \%$ of cases occurs in patients without identified risk factors. The sudden death of an apparently healthy person - their age notwithstanding - has a devastating emotional effect on others. Many cardiac arrests occur at home or in public places and patients are suddenly transported to the nearest ER under basic life support (BLS) maneuvers or sometimes the impending cardiac arrest summons people where the event occurs.

Many authors tackled the issue of the appropriateness of CPR in the elderly, most of all when considering doubts on the efficacy and of the post-CPR quality of life.

Currently, in many countries the possibility of stating an a priori DNR order often generates more ethical problems than giving operative solutions when facing a cardiac arrest. ${ }^{\mathrm{vi}}$ Only $<25 \%$ of decisions about CPR or DNR orders seems to be unequivocally expressed by patients after given appropriate information. ${ }^{7}$ In this perspective, the ER setting offers a unique condition where anamnestic data are usually lacking, saving time is essential and decisions cannot be delayed so that it is the physician's ethical and professional evaluation to rule out the aggressive or non-aggressive intervention while the boundary between appropriate care and therapeutic obstinacy is often difficult to be set. Also, it should always be kept in mind that there is no clear evidence that patients who died after choosing DNR orders would not have survived, if CPR had been attempted. ${ }^{8,9}$ On the other hand, healthcare providers performing CPR in ER on elderly patients often find themselves in morally distressing circumstances because of anecdotal knowledge of poor outcomes and the patient's frustration as to resuscitation procedures. ${ }^{10}$ In our study, we evaluated rough data from records of cardiac arrest occurred in the ER of a main hospital in Rome, Italy. We chose not to evaluate some data like the time spent in the resuscitation effort because of the bias that could be introduced by non-medical matters. We focused our attention on the time spent in the ER and did not follow the outcome of the patients after the admission to the ICU.

As it could be expected, the age range of the patients arriving at the ER with cardiac arrest is very wide (0-96) and, interestingly, the mean age is about 70 and do not significantly vary among the 4 outcome groups.
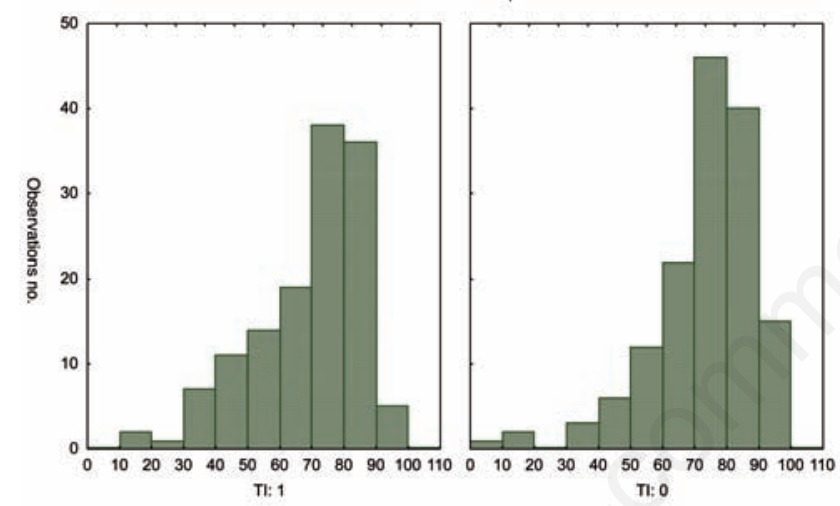

Figure 4. Comparison of different age distributions with tracheal intubation procedure (intubated: left panel; or not intubated: right panel).

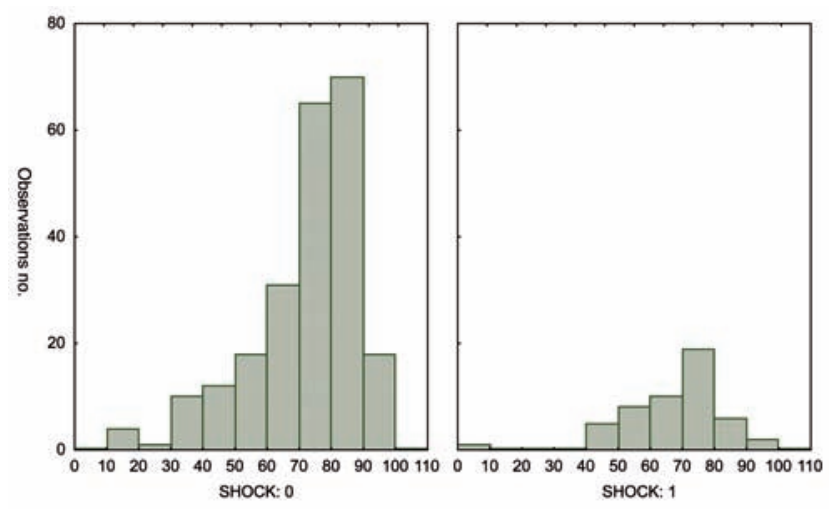

Figure 5. Comparison of different age distributions with erogation of direct current shock. Direct current shock non deliverd: left panel; direct shock delivered: right panel.

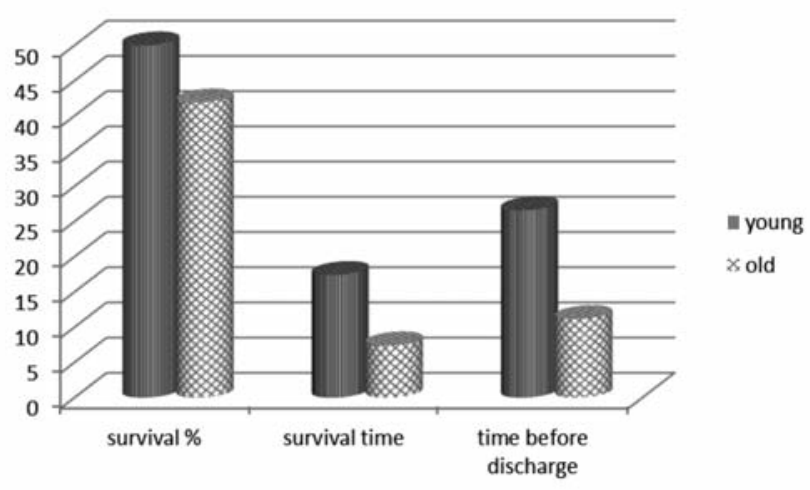

Figure 7. Mean survival time and hospital staying of discharged patients both young and old. 
The overall survival to cardiac arrest observed in our population is close to the lowest limit reported in the literature ${ }^{11,12}$ ( 7 to $26 \%$ ), but, for elder population, it appears to be lower than that reported by Abbo et al. and Ehlenbach. ${ }^{13,14}$ Old people seem to have the same chance of surviving a cardiac arrest than young people, although the differences in the oro-TI show that there is probably a less aggressive behavior in resuscitators in older patients.

Other factors like comorbidity can influence the outcome of a cardiac arrest in the elderly.

\section{Conclusions}

The results of the present study demonstrate that age itself does not appear to be an independent, unfavorable prognostic factor for the outcome after CPR and that older people must be offered the same chance of surviving a cardiac arrest as youngsters.

\section{References}

1. Granja C, Cabral G, Pinto AT, Costa-
Pereira A. Quality of life 6-months after cardiac arrest. Resuscitation 2002;55:3744.

2. Berger R, Kelley M. Survival after in-hospital cardiopulmonary arrest of non-critically ill patients. A prospective study. Chest 1994;106:872-9.

3. Ballew KA, Philbrick JT, Caven DE, et al. Predictors of survival following in-hospital cardiopulmonary resuscitation. A moving target. Arch Intern Med 1994;154:2426-32.

4. de Vos R, Koster RW, De Haan RJ, et al. Inhospital cardiopulmonary resuscitation: prearrest morbidity and outcome. Arch Intern Med 1999;159:845-50.

5. Deasy C, Bray JE, Smith K, et al. Out-of hospital cardiac arrests in the older age groups in Melbourne, Australia. Resuscitation 2011;82:398-403.

6. Somogyi-Zalud E, Zhong Z, Hamel MB, Lynn J. The use of life-sustaining treatments in hospitalized persons aged 80 and older. J Am Geriatr Soc 2002;50:930-4.

7. Rabkin MT, Gillerman G, Rice NR. Orders not to resuscitate. New Engl J Med 1976;295:364-6.

8. Quill TE, Bennett NM. The effects of a hospital policy and state legislation on resuscitation orders for geriatric patients.
Arch Intern Med 1992;152:569-72.

9. Stephens RL. "Do not resuscitate" orders: ensuring the patient's participation. JAMA-J Am Med Assoc 1986;255:240-1.

10. Robinson EM. An ethical analysis of cardiopulmonary resuscitation for elders in acute care. AACN Clin Issues 2002;13:13244.

11. Tresch D, Heudebert G, Kutty K, et al. Cardiopulmonary resuscitation in elderly patients hospitalized in the 1990s: a favorable outcome. J Am Geriatr Soc 1994;42: 137-41.

12. Ebell MH, Becker LA, Barry HC, Hagen M. Survival after in-hospital cardiopulmonary resuscitation: a meta-analysis. J Gen Intern Med 1998;13:805-16.

13. Abbo ED, Yuen TC, Buhrmester L, et al. Cardiopulmonary resuscitation outcomes in hospitalized community-dwelling individuals and nursing home residents based on activities of daily living. J Am Geriatr Soc 2013;61:34-9.

14. Ehlenbach WJ, Barnato AE, Curtis JR, et al. Epidemiologic study of in-hospital cardiopulmonary resuscitation in the elderly. New Engl J Med 2009;361:22-31. 